Elsevier Editorial System(tm) for Physics Letters A

Manuscript Draft

Manuscript Number:

Title: Application of a modified rational harmonic balance method for a class of strongly nonlinear oscillators

Article Type: Letter

Section/Category: Mathematical physics

Keywords: Nonlinear oscillators; Approximate solutions; Rational harmonic balance method.

Corresponding Author: Dr. Augusto Beléndez, PhD

Corresponding Author's Institution: University of Alicante

First Author: Augusto Beléndez, PhD

Order of Authors: Augusto Beléndez, PhD; Encarnación Gimeno; Mariela L. Álvarez; David I. Méndez; Antonio Hernández

Manuscript Region of Origin: 


\title{
Application of a modified rational harmonic balance method for a class of strongly nonlinear oscillators
}

\author{
A. Beléndez, E. Gimeno, M. L. Álvarez, D. I. Méndez and A. Hernández
}

Departamento de Física, Ingeniería de Sistemas y Teoría de la Señal.

Universidad de Alicante.

Apartado 99. E-03080 Alicante. SPAIN

E-mail: a.belendez@ua.es

\author{
Corresponding author: A. Beléndez \\ Phone: +34-6-5903651 \\ Fax: +34-6-5903464
}




\begin{abstract}
An analytical approximate technique for conservative nonlinear oscillators is proposed. This method is a modification of the rational harmonic balance method in which analytical approximate solutions have rational form. This approach gives us the frequency of the motion as a function of the amplitude of oscillation. We find that this method works very well for the whole range of parameters, and excellent agreement of the approximate frequencies with the exact one has been demonstrated and discussed. The most significant features of this method are its simplicity and its excellent accuracy for the whole range of oscillation amplitude values and the results reveal that this technique is very effective and convenient for solving conservative truly nonlinear oscillatory systems with complex nonlinearities.
\end{abstract}

Keywords: Nonlinear oscillators; Approximate solutions; Rational harmonic balance method.

\title{
1. Introduction
}

Nonlinear oscillation in physics and applied mathematics has been a topic to intensive research for many years. There are a large variety of approximate methods commonly used for solving nonlinear oscillatory systems including perturbations [1-4], parameterexpansion [5, 6], variational [7], variational iteration [8], homotopy perturbation [9-12], harmonic balance [1, 13-17] methods, etc. Surveys of the literature with numerous references and useful bibliography and a review of these methods can be found in detail in [2] and [18]. In this paper we apply a modified generalized, rational harmonic balance method to obtain analytic approximate solutions for a nonlinear oscillator for which the restoring force has a rational expression of the displacement. This method can be applied to nonlinear oscillatory systems where the nonlinear terms are not small and no perturbation parameter is required. In this method the approximate solution obtained 
approximates all of the harmonics in the exact solution [19], whereas the usual harmonic balance techniques provide an approximation to only the lowest harmonic components. In an attempt to provide better solution methodology a modification in this technique is proposed.

\section{Formulation and solution method}

To apply the modify rational harmonic balance method to we consider the following nonlinear oscillator

$$
\frac{\mathrm{d}^{2} x}{\mathrm{~d} t^{2}}+\frac{x}{1+x^{2}}=0
$$

with initial conditions

$$
x(0)=A \quad \text { and } \quad \frac{\mathrm{d} x}{\mathrm{~d} t}(0)=0
$$

Eq. (1) is an example of a conservative nonlinear oscillatory system in which the restoring force has a rational form

$$
\frac{\mathrm{d}^{2} x}{\mathrm{~d} t^{2}}=F(x), \quad F(x)=-\frac{x}{1+x^{2}}
$$

All the motions corresponding to Eq. (1) are periodic [18] and the system will oscillates between symmetric bounds $[-A, A]$, and the frequency and corresponding periodic solution of the oscillator are dependent on the amplitude $A$. Note that for $x$, respectively, small and large, Eq. (1) becomes

$$
x \text { small } \frac{\mathrm{d}^{2} x}{\mathrm{~d} t^{2}}+x \approx 0, \quad x \text { large } \quad \frac{\mathrm{d}^{2} x}{\mathrm{~d} t^{2}}+\frac{1}{x} \approx 0
$$

Thus, for $x$ small, the equation of motion approximates that of a linear harmonic oscillator and its angular frequency is $\omega \approx 1$ for small $A$. However, for large $x$, the equation of motion is that of a truly nonlinear oscillator in which the restoring force is 
inversely proportional to the dependent variable and $\omega \approx \sqrt{2 \pi} / 2 A=1.25331 / A$ [20-22], which tends to zero when $A$ decreases. Consequently the angular frequency $\omega$ increases from 1 to $1.25331 / A$ as the initial value of $x(0)=A$ increases. The main purpose of this letter is to construct an analytical approximation to the solution of Eq. (1) using a modified rational harmonic balance method (RHBM) introduced by Beléndez et al. in ref. [22] and which has been applied for truly conservative nonlinear oscillators with good results.

To solve Eq. (1) by the modified RHBM, a new independent variable $\tau=\omega t$ is introduced. Then Eqs. (1) and (2) can be rewritten as

$$
\begin{gathered}
\omega^{2} \frac{\mathrm{d}^{2} x(\tau)}{\mathrm{d} \tau^{2}}+\frac{x(\tau)}{1+x^{2}(\tau)}=0 \\
x(0)=A, \quad \frac{\mathrm{d} x}{\mathrm{~d} \tau}(0)=0
\end{gathered}
$$

The new independent variable is chosen in such a way that the solution of Eq. (5) is a periodic function of $\tau$ of period $2 \pi$. The corresponding frequency of the nonlinear oscillator is $\omega$ and it is a function of the amplitude $A$.

Following the lowest order harmonic balance approximation, we set $x_{1}(\tau)=A \cos \tau$ which satisfies the initial conditions in Eq. (6). Substituting $x_{1}(\tau)$ into Eq. (5) we obtain

$$
-\omega^{2} A \cos \tau+\frac{A \cos \tau}{1+A^{2} \cos ^{2} \tau}=0
$$

We can do the following Fourier series expansion

$$
\frac{A \cos \tau}{1+A^{2} \cos ^{2} \tau}=\sum_{n=0}^{\infty} a_{2 n+1} \cos [(2 n+1) \tau]
$$

where

$$
a_{2 n+1}=\frac{4}{\pi} \int_{0}^{\pi / 2} \frac{A \cos \tau}{1+A^{2} \cos ^{2} \tau} \cos [(2 n+1) \tau] d \tau
$$


Substituting Eq. (8) into Eq. (7) gives

$$
\left(-\omega^{2} A+a_{1}\right) \cos \tau+H O H=0
$$

where $\mathrm{HOH}$ stands for higher-order harmonics. Setting the coefficient of $\cos \tau$ to zero allows the determination of the first approximation to the frequency in terms of $A$

$$
\omega_{1}(A)=\sqrt{\frac{a_{1}}{A}}=\sqrt{\frac{2}{A^{2}}-\frac{2}{A^{2} \sqrt{1+A^{2}}}}
$$

In order to determine an improved approximation we use a rational form given by the following expression $[1,19]$

$$
x_{2}(\tau)=\frac{A_{1} \cos \tau}{1+B_{2} \cos 2 \tau}
$$

In this equation $A_{1}, B_{2}$ and $\omega$ are to be determined as functions of the initial conditions expressed in Eq. (6) and $\left|B_{2}\right|<1$. From Eq. (6) we obtain $A_{1}=\left(1+B_{2}\right) A$ and Eq. (12) can be rewritten as follows

$$
x_{2}(\tau)=\frac{\left(1+B_{2}\right) A \cos \tau}{1+B_{2} \cos 2 \tau}
$$

Substituting Eq. (13) into Eq. (5) leads to

$$
\begin{aligned}
& -\omega^{2} \frac{A\left(1+B_{2}\right) \cos \tau}{1+B_{2} \cos 2 \tau}+\omega^{2} \frac{4 A B_{2}\left(1+B_{2}\right) \cos \tau \cos 2 \tau-4 A B_{2}\left(1+B_{2}\right) \sin \tau \sin 2 \tau}{\left(1+B_{2} \cos 2 \tau\right)^{2}} \\
& +\omega^{2} \frac{8 A B_{2}^{2}\left(1+B_{2}\right) \cos \tau \sin ^{2} 2 \tau}{\left(1+B_{2} \cos 2 \tau\right)^{3}}+\frac{A\left(1+B_{2}\right)\left(1+B_{2} \cos 2 \tau\right) \cos \tau}{\left(1+B_{2} \cos 2 \tau\right)^{2}+A^{2}\left(1+B_{2}\right)^{2} \cos ^{2} \tau}=0
\end{aligned}
$$

Eq. (14) can be written as follows 


$$
F\left(A, B_{2}, \omega, \tau\right)=0
$$

As $\left|B_{2}\right|<1$ we can do the following series expansion

$$
F\left(A, B_{2}, \omega, \tau\right)=\sum_{n=0}^{\infty} f_{n}(A, \omega, \tau) B_{2}^{n}
$$

where

$$
f_{n}(A, \omega, \tau)=\frac{1}{n !}\left(\frac{\partial^{n} F\left(A, B_{2}, \omega, \tau\right)}{\partial B_{2}^{n}}\right)_{B_{2}=0}
$$

Before applying the harmonic balance method to Eq. (14) and we consider the following approximation in Eqs. (15)-(17)

$$
F\left(A, B_{2}, \omega, \tau\right) \approx f_{0}(A, \omega, \tau)+f_{1}(A, \omega, \tau) B_{2}+f_{2}(A, \omega, \tau) B_{2}^{2}=0
$$

Expanding Eq. (18) in a trigonometric series yields

$$
H_{1}\left(A, B_{2}, \omega\right) \cos \tau+H_{3}\left(A, B_{2}, \omega\right) \cos 3 \tau+H O H
$$

where

$$
\begin{gathered}
H_{1}\left(A, B_{2}, \omega\right)=\frac{4}{\pi} \int_{0}^{\pi / 2}\left(f_{0}+f_{1} B_{2}+f_{2} B_{2}^{2}\right) \cos \tau d \tau \\
H_{3}\left(A, B_{2}, \omega\right)=\frac{4}{\pi} \int_{0}^{\pi / 2}\left(f_{0}+f_{1} B_{2}+f_{2} B_{2}^{2}\right) \cos 3 \tau d \tau
\end{gathered}
$$

Setting the coefficients of $\cos \tau$ and $\cos 3 \tau$ to zero in Eq. (19) we can obtain $B_{2}$ and the second order approximate frequency $\omega$ as a function of $A$. From Eqs. (14)-(21) we obtain 


$$
\begin{aligned}
H_{1}\left(A, B_{2}, \omega\right) & =\frac{1}{2 A^{6}}\left[160 B_{2}^{2}+24 A^{2} B_{2}\left(4 B_{2}-1\right)+4 A^{4}\left(B_{2}^{2}-B_{2}+1\right)-A^{6}\left(B_{2}+2\right) \omega^{2}\right] \\
& -\frac{2}{A^{6} J(A)}\left[40 B_{2}^{2}+2 A^{2} B_{2}\left(22 B_{2}-3\right)+A^{4}\left(8 B_{2}^{2}-4 B_{2}+1\right)\right]=0 \\
H_{3}\left(A, B_{2}, \omega\right) & =-\frac{1}{4 A^{8}}\left[2688 B_{2}^{2}+320 A^{2} B_{2}\left(10 B_{2}-1\right)+8 A^{6}\left(2 B_{2}^{2}-2 B_{2}+1\right)+16 A^{4} B_{2}\left(B_{2}+2\right) \omega^{2}\right] \\
+ & \frac{2}{A^{8} J(A)}\left[336 B_{2}^{2}+8 A^{2} B_{2}\left(71 B_{2}-5\right)+A^{4}\left(260 B_{2}^{2}-50 B_{2}+4\right)+3 A^{6}\left(8 B_{2}^{2}-4 B_{2}+1\right)\right]=0
\end{aligned}
$$

where

$$
J(A)=\sqrt{1+A^{2}}
$$

Solving Eq. (22a) for $\omega$ yields

$$
\omega_{2}(A)=\frac{2 \sqrt{\left[A^{4}-\left(6 A^{2}+A^{4}\right) B_{2}+\left(40+24 A^{2}+A^{4}\right) B_{2}^{2}\right] J(A)-A^{4}-\left(6 A^{2}+4 A^{4}\right) B_{2}+\left(40+44 A^{2}+8 A^{4}\right) B_{2}^{2}}}{A^{3} \sqrt{\left(2+B_{2}\right) J(A)}}
$$

and substituting Eq. (24) into Eq. (22b) gives the following cubic equation which must be solved to obtain $B_{2}$

$$
\begin{aligned}
24 A^{6} & -8 A^{6} J(A)-32 A^{4}(J(A)-1) \\
+ & {\left[-320 A^{2}-36 A^{6}+320 A^{2} J(A)+36 A^{6} J(A)+16 A^{6}(J(A)-6)+80 A^{4}(3 J(A)-5)\right] B_{2} } \\
+ & {\left[216 A^{4}-216 A^{4} J(A)-16 A^{6}(J(A)-12)-36 A^{6}(J(A)-4)\right.} \\
& \left.-2688(J(A)-1)-64 A^{2}(50 J(A)-71)-16 A^{4}(51 J(A)-130)\right] B_{2}^{2} \\
+ & {\left[36 A^{6}(J(A)-8)+1440 A^{2}(J(A)-1)+144 A^{4}(6 J(A)-11)\right] B_{2}^{3}=0 }
\end{aligned}
$$

As we can see in Eq. (24), for $B_{2}=0$, the approximate frequency for the first-order harmonic balance approximation is obtained

$$
\omega_{1}(A)=\sqrt{\frac{2}{A^{2}}-\frac{2}{A^{2} \sqrt{1+A^{2}}}}
$$


In Figure 1 we have plotted $B_{2}$ as a function of $A$. Furthermore, from Eq. (25) we can obtain

$$
\lim _{A \rightarrow \infty} B_{2}=0.1831313
$$

Therefore, the second approximation to the periodic solution of the nonlinear oscillator is given bay the following equation

$$
\frac{x_{2}(t)}{A}=\frac{\left(1+B_{2}\right) \cos \omega_{2} t}{1+B_{2} \cos 2 \omega_{2} t}
$$

This periodic solution has the following Fourier series expansion

$$
\frac{x_{2}(t)}{A}=\sum_{n=0}^{\infty} a_{2 n+1} \cos \left[(2 n+1) \omega_{2} t\right]
$$

where

$$
a_{2 n+1}=\frac{4}{\pi} \int_{0}^{\pi / 2} \frac{\left(1+B_{2}\right) \cos \tau}{1+B_{2} \cos 2 \tau} \cos [(2 n+1) \tau] d \tau
$$

As we can see, Eq. (29) gives an expression that approximates all of the harmonics in the exact solution whereas the usual harmonic balancing techniques provide and approximation to only the lowest harmonic components.

\section{Discussion}

We illustrate the accuracy of the modified approach by comparing the approximate solutions previously obtained with the exact frequency $\omega_{e}$. Direct integration of Eq. (1) yields the exact frequency as

$$
\omega_{e}(A)=\frac{\pi}{2 A}\left(\int_{0}^{1} \frac{d u}{\sqrt{\log \left[\left(1+A^{2}\right) /\left(1+A^{2} u^{2}\right)\right.}}\right)^{-1}
$$


In Figure 2 we plotted the relative error of the approximate frequency $\omega_{2}(A)$ (Eq. (24)) as a function of $A$. The results are valid for the complete range of oscillation amplitude, including the limiting cases of amplitude approaching zero and infinity. As we can see from Figure 2, the exact and approximate results are very similar and the relative error tends to $0.40 \%$ when $A$ tends to infinity. As we pointed out in the previous section, when $A$ tends to infinity the behaviour of the oscillator is such as a nonlinear oscillator in which the restoring force is inversely proportional to the dependent variable [20-22]. These results are an indication of the accuracy of the proposed modified rational harmonic balance method as applied to this particular problem and show that it provides an excellent approximation to the solution of Eq. (1).

For small values of the amplitude $A$ it is possible to take into account the following approximation

$$
\begin{aligned}
\omega_{e}(A) & \approx 2 \pi\left[\int_{0}^{1} \frac{\mathrm{d} u}{96 \sqrt{1-u^{2}}}\left(384+96\left(1+u^{2}\right) A^{2}-4\left(7-2 u^{2}+7 u^{4}\right) A^{4}+3\left(5-u^{2}-u^{4}+5 u^{6}\right) A^{6}+\ldots\right)\right]^{-1} \\
& =\frac{2048}{2048+768 A^{2}-184 A^{4}+91 A^{6}+\ldots}
\end{aligned}
$$

and the power series expansion of the exact frequency, $\omega_{e}(A)$, for $A$ small is

$$
\omega_{e}(A) \approx 1-\frac{3}{8} A^{2}+\frac{59}{256} A^{4}-\frac{337}{2048} A^{6}+\ldots
$$

For small values of $A$ it is also possible to do the power series expansion of the solution of Eq. (25) and we obtain (we omit the expression of the exact solution for $B_{2}$ and we also include its power series expansion for small values of $A$ )

$$
B_{2}(A) \approx \frac{1}{16} A^{2}-\frac{17}{512} A^{4}+\frac{83}{4096} A^{6}+\ldots
$$

Substituting Eq. (34) into Eq. (24) and doing the power series expansion for small values of $A$, the following equation can be obtained 


$$
\omega_{2}(A) \approx 1-\frac{3}{8} A^{2}+\frac{59}{256} A^{4}-\frac{341}{2048} A^{6}+\ldots
$$

As we can see, in the expansion of the angular frequency obtained in this paper, $\omega_{2}(A)$ (Eq. (35)), the first three terms are the same as the first three terms of the equation obtained in the power-series expansion of the exact angular frequency, $\omega_{e}$ (Eq. (33)), and the relative error for the fourth term is $<1.2 \%$.

For very large values of the amplitude $A$ it is possible to take into account the following power series expansion for Eq. (31)

$$
\omega_{e}(A) \approx 2 \pi\left[\int_{0}^{1} \frac{A \mathrm{~d} u}{\sqrt{\log \left(1 / u^{2}\right)}}\right]^{-1} \approx \frac{1.25331}{A}
$$

For very large values of $A$ it is also possible to do the power series expansion of the solution of Eq. (25) and we obtain

$$
B_{2} \approx \frac{13}{27}-\frac{182}{27}\left(\frac{2}{-4921+189 \sqrt{1535}}\right)^{1 / 3}+\frac{1}{27}\left[\frac{1}{2}(-4921+189 \sqrt{1535})\right]^{1 / 3}+\ldots \approx 0.1831313+\ldots
$$

Substituting Eq. (37) into Eq. (24) and doing the power series expansion for very large values of $A$, the following equation can be obtained

$$
\omega_{2}(A) \approx \frac{1.24825}{A}+\ldots
$$

If we compare Eqs. (36) and (38) we can see that the relative error for the approximate frequency is $0.40 \%$ for very large values of $A$.

The normalized periodic exact solution, $x_{e} / A$, achieved using Eq. (1) and the proposed second-order approximate solution, $x_{2} / A$ (Eq. (28)) are plotted in Figures 3, 4 and 5 for $A=1,10$ and 100, respectively. In these figures $h$ is defined as $h=t / T_{e}$. These figures show that Eqs. (24) and (28) can provide high accurate approximations to the exact frequency and the exact periodic solution. 


\section{Conclusions}

In this study, we applied a modified rational harmonic balance method to approximately solve a nonlinear oscillator in which the restoring force has a rational expression. The major conclusion is that this scheme provides excellent approximations to the solution of this nonlinear system with high accuracy and, in particular, the results for the oscillator analyzed are valid for the whole solution domain. The analytical representations obtained using this technique give excellent approximations to the exact solutions for the whole range of values of oscillation amplitude. In summary, this modified generalized harmonic balance method is very simple in its principle, and it can be used to solve other conservative nonlinear oscillators with complex nonlinearities.

\section{References}

[1] R. E. Mickens, Oscillations in Planar Dynamics Systems (World Scientific, Singapore 1996).

[2] J. H. He, Non-perturbative methods for strongly nonlinear problems (dissertation.de-Verlag im Internet $\mathrm{GmbH}$, Berlin 2006).

[3] P. Amore, A. Raya and F. M. Fernández, "Alternative perturbation approaches in classical mechanics”, Eur. J. Phys. 26, 1057-1063 (2005).

[4] J. H. He, "Modified Lindstedt-Poincare methods for some non-linear oscillations. Part I: expansion of a constant”, Int. J. Non-linear Mech. 37, 309-314 (2002).

[5] M. T. Darvishi, A. Karami and B.C.Shin, "Application of He's parameterexpansion method for oscillators with smooth odd nonlinearities", Phys. Lett. A. (2008) doi:10.1016/j.physleta.2008.06.058.

[6] S. Q. Wang and J. H. He, "Nonlinear oscillator with discontinuity by parameterexpansion method", Chaos, Solitons \& Fractals 35, 688-691 (2008).

[7] J. H. He, "Variational approach for nonlinear oscillators", Chaos, Solitons \& Fractals 34, 1430-1439 (2007). 
[8] J. H. He and X. H. Wu, "Construction of solitary solution and compacton-like solution by variational iteration method", Chaos, Solitons \& Fractals 29, 108-113 (2006).

[9] J. H. He, "Homotopy perturbation method for bifurcation on nonlinear problems", Int. J. Non-linear Sci. Numer. Simulat. 6, 207-208 (2005).

[10] A. Beléndez, A. Hernández, T. Beléndez, E. Fernández, M. L. Álvarez and C. Neipp, “Application of He's homotopy perturbation method to the Duffingharmonic oscillator”, Int. J. Non-linear Sci. Numer. Simulat. 8, 79-88 (2007).

[11] M. Gorji, D. D. Ganji and S. Soleimani, "New application of He's homotopy perturbation method", Int. J. Non-linear Sci. Numer. Simulat. 8, 319-328 (2007).

[12] A. Beléndez, A. Hernández, T. Beléndez, C. Neipp and A. Márquez, “Application of the homotopy perturbation method to the nonlinear pendulum", Eur. J. Phys. 28, 93-104 (2007).

[13] A. Beléndez, A. Hernández, A. Márquez, T. Beléndez and C. Neipp, “Analytical approximations for the period of a simple pendulum", Eur. J. Phys. 27, 539-551 (2006).

[14] M. Rafei y D. D. Ganji, "Explicit solutions of Helmhotlz equation and fifth-order $\mathrm{KdV}$ equation using homotopy perturbation method”, Int. J. Non-linear Sci. Numer. Simulat. 7, 321-328 (2006).

[15] H. Hu and J. H. Tang, "Solution of a Duffing-harmonic oscillator by the method of harmonic balance", J. Sound Vib. 294, 637-639 (2006).

[16] C. W. Lim and B. S. Wu, "A new analytical approach to the Duffing-harmonic oscillator", Phys. Lett. A 311, 365-373 (2003).

[17] A. Beléndez, A. Hernández, T. Beléndez, M. L. Álvarez, S. Gallego, M. Ortuño and C. Neipp, "Application of the harmonic balance method to a nonlinear oscillator typified by a mass attached to a stretched wire", J. Sound Vib. 302, 1018-1029 (2007).

[18] J. H. He, "Some asymptotic methods for strongly nonlinear equations", Int. J. Mod. Phys. B 20, 1141-1199 (2006). 
[19] R. E. Mickens and D. Semwogerere, "Fourier analysis of a rational harmonic balance approximation for periodic solutions", J. Sound Vib. 195, 528-530 (1996).

[20] A. Beléndez, D.I. Méndez, T. Beléndez, A. Hernández, M.L. Álvarez, "Harmonic balance approaches to the nonlinear oscillators in which the restoring force is inversely proportional to the dependent variable", J. Sound Vib. 314, 775-782 (2008).

[21] J. I. Ramos, “An artificial parameter Linstedt-Poincaré method for the periodic solutions of nonlinear oscillators in which the restoring term force is inversely proportional to the dependent variable", J. Sound Vib. doi:10.1016/j.jsv. 2008.05.004 (2008)

[22] A. Beléndez, E. Gimeno, E. Fernández, D. I. Méndez and M. L. Álvarez, "Accurate approximate solution to nonlinear oscillators in which the restoring force is inversely proportional to the dependent variable", Phys. Scr. 77, 065004 (2008). 


\section{FIGURE CAPTIONS}

Figure 1.- $\quad$ Parameter $B_{2}$ as a function of $A$.

Figure 2.- Relative error for the approximate frequency $\omega_{2}$ as a function of $A$.

Figure 3.- Comparison of the normalized second order approximate solution $(\mathrm{O}$ and dashed line) with the exact solution (continuous line) for $A=1$.

Figure 4.- Comparison of the normalized second order approximate solution ( $\mathrm{O}$ and dashed line) with the exact solution (continuous line) for $A=10$.

Figure 5.- Comparison of the normalized second order approximate solution ( $\mathrm{O}$ and dashed line) with the exact solution (continuous line) for $A=100$. 




FIGURE 1

Figure 1 




FIGURE 2

Figure 2 


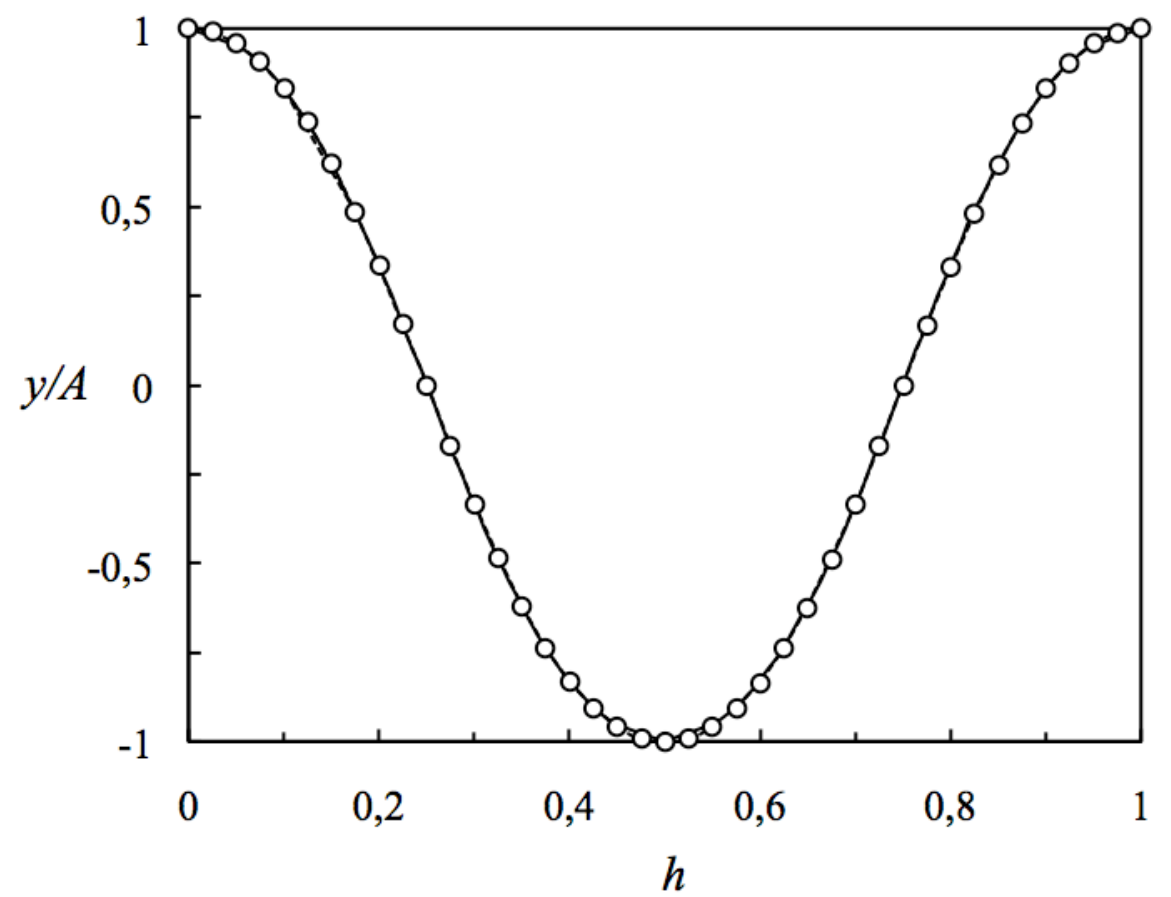

FIGURE 3 
Figure 4

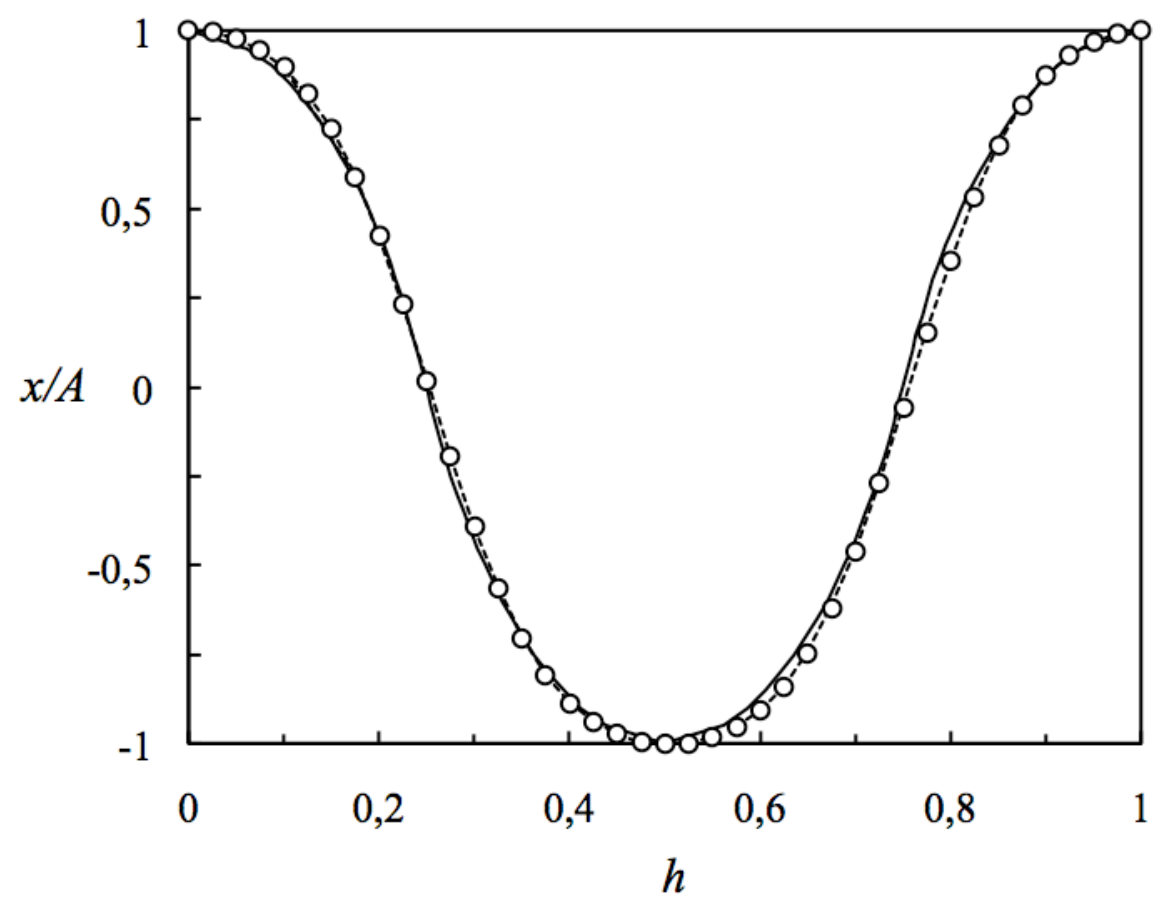

FIGURE 4 


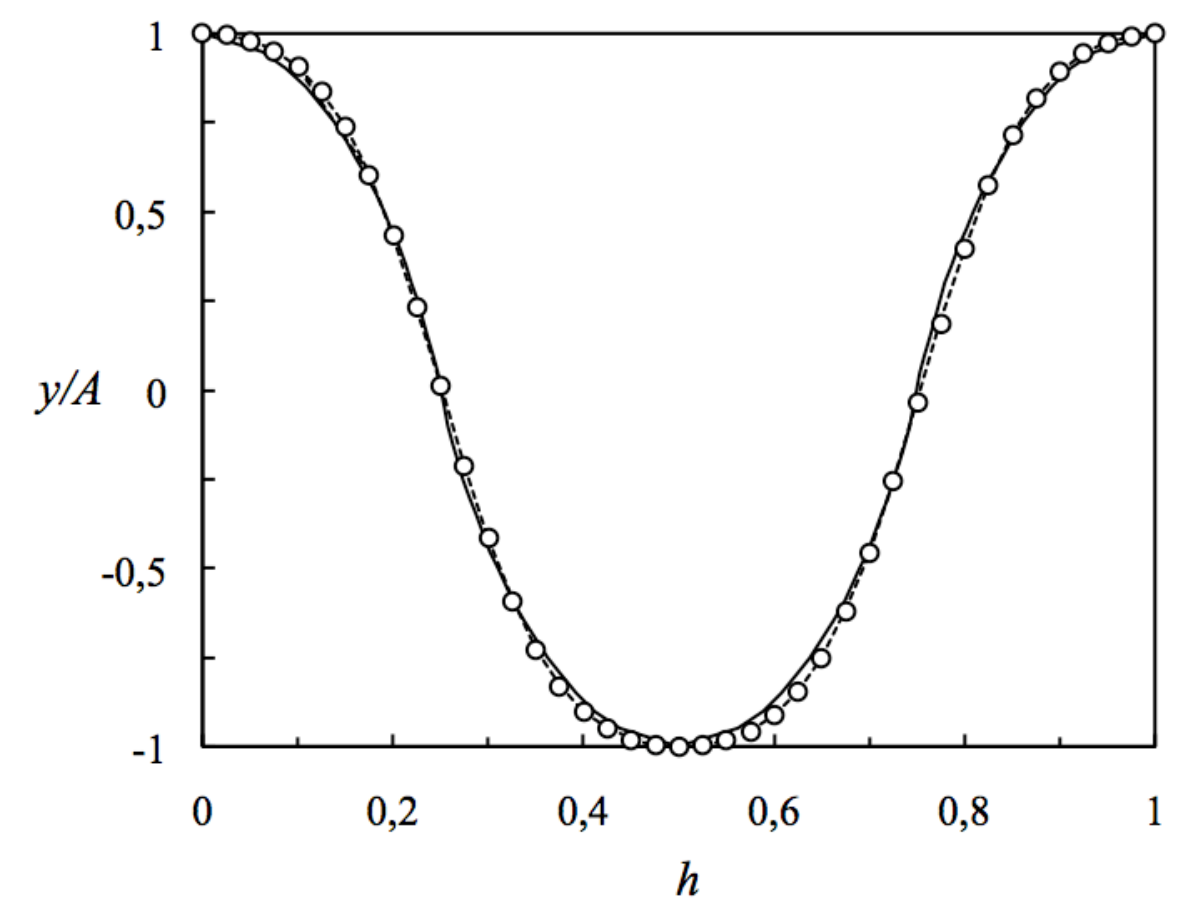

FIGURE 5

Figure 5 